\title{
Primär sklerosierende Cholangitis: Entwicklung neuer Therapieansätze
}

Bei der primär sklerosierenden Cholangitis (PSC) gibt es bislang keine etablierte medikamentöse Therapie. Das könnte sich in absehbarer Zukunft ändern. Denn derzeit sind mehrere neue Behandlungsansätze in Entwicklung.

Wird eine PSC diagnostiziert, so liegt die mittlere Lebenserwartung des Patienten bei nur noch zehn bis zwölf Jahren. Das zeigt den dringenden Bedarf für therapeutische Fortschritte bei der cholestatischen Lebererkrankung. Bislang steht dabei die endoskopische Therapie im Vordergrund. Behandelt wird ferner mit Ursodesoxycholsäure (UDCA, z.B. Ursofalk ${ }^{\bowtie}$ ), berichtete Prof. Dr. Michael Trauner aus Wien bei einem Symposium der Falk Foundation e.V. beim 121. Jahreskongress der Deutschen Gesellschaft für Innere Medizin in Mannheim.

Als Hoffnungsträger nannte der Hepatologe norUDCA, eine Modifikation der
UDCA, die nicht der enterohepatischen Zirkulation unterliegt, sondern ein cholehepatisches Shunting durchmacht und sich dadurch stärker in der Leber und im Gallengangsepithel anreichert. „Das scheint sich in einer höheren Effektivität niederzuschlagen“, so Trauner.

\section{Neue Option wird in europäischer Studie geprüft}

Im Tiermodell ist norUDCA in der Lage, die PSC zu heilen und auch die ersten Phase I-Studien belegen eine gute antiinflammatorische, antiproliferative und antifibrotische Wirksamkeit. „Die neue Option wird derzeit in einer europäischen multizentrischen Phase II-Studie bei mehr als 160 Patienten mit PSC geprüft“, so Trauner.

Bestätigen sich die günstigen Vorbefunde, so ist zu hoffen, dass sich durch norUDCA auch bei der primär biliären Zirrhose (PBC) Therapiefortschritte erzielen lassen. Bei beiden cholestatischen Krankheitsbildern, der PSC und der PBC, bei der UDCA derzeit weltweit die Standardtherapie darstellt, gibt es vermutlich in absehbarer Zukunft weitere Neuerungen. So wird intensiv an der Entwicklung spezifischer Liganden für den nukleären Gallensäurerezeptor FXR (Farnesoid X Rezeptor) gearbeitet. Eine weitere potenzielle Neuerung ist die Obeticholsäure, eine chemische Modifikation der Chenodesoxycholsäure, bei der in ersten Studien eine Besserung der Cholestase-Parameter bei der PBC dokumentiert wurde.

\section{Hoffnung auf Fortschritte beim Gallengangskarzinom}

Das cholangiozelluläre Karzinom (CCC) gehört zwar zu den seltenen Tumoren, doch ist seit rund zehn Jahren ein deutlicher Anstieg der Inzidenz und auch der Mortalität zu verzeichnen. „Weltweit gehen pro Jahr rund 200.000 Todesfälle auf das Konto dieser Erkrankung“, berichtete Prof. Dr. Nisar P. Malek aus Tübingen. Auch in Deutschland nimmt die Häufigkeit des CCC zu. Die Mortalität des intrahepatischen CCC ist in den vergangenen Jahren dabei um rund $400 \%$ gestiegen, während die Häufigkeit der extrahepati- schen und Gallenblasen-Karzinome in etwa konstant geblieben ist.

\section{0-90\% der Karzinome sind nicht primär operabel}

Als wichtigste Therapieoption nannte Malek die Resektion, allerdings sind 50-90\% der CCC nicht primär operabel.

Die Chemotherapie ist der „Best Supportive Care" überlegen, zeigt jedoch ebenfalls kaum überzeugende Therapieerfolge, da der Tumor meist erst im fortgeschrittenen Stadium entdeckt wird. So

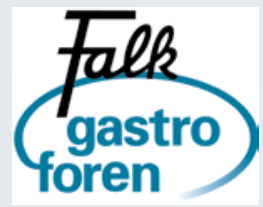

Besuchen Sie das nächste Falk Gastro Forum „Chronische Erkrankungen des Verdauungstrakts: Heilung durch individualisierte Therapie?“ am Samstag, den 4. Juli 2015 in Hannover; Infos: www.drfalkpharma.de/veranstaltungen

liegt das 5-Jahres-Überleben bei nur 5-10\%.

Hoffnungen auf eine Besserung der Überlebenswahrscheinlichkeit gründen sich derzeit vor allem auf die Entwicklung molekularer Therapieregime. Es wurden verschiedene genetische Veränderungen beim CCC identifiziert, die nun Ausgangspunkt für Studien sein können. Im Fokus stehen dabei der bei einem Teil der Patienten überexprimierte Wachstumsfaktor EGFR, der cMET-Signalweg und der Notch-Signalweg, der bei der Mehrzahl der Patienten verändert ist. Außerdem sind immuntherapeutische Therapieansätze in Entwicklung.

(CV)

21. Symposium Aktuelle Hepatologie 2015 der Falk Foundation e.V. am 18. April 2015 beim 121. Kongress der Deutschen Gesellschaft für Innere Medizin in Mannheim 